\title{
Correlation Between Cytologic Results and Thyroid Autoantibodies, Calcitonin, and Thyroid Function Tests in Patients with Thyroid Nodules
}

\author{
Pinar Karakaya, Ahmet Cem Dural, Cevher Akarsu, Ozlem Soylukbiricik, Abdulbaki Kumbasar \\ Bahar Ozdemir, Bulent Yaprak, Meral Mert
}

Bakırköy Dr. Sadi Konuk Research And Training Hospital Turkey

\begin{abstract}
Background: Fine needle aspiration biopsy (FNAB) is currently a widely accepted screening procedure in diagnosis of thyroid nodules, there has been confusion related to diagnostic terminology in the assessment of samples. This confusion has been caused by multiple category names, descriptive reports without assigning to a category, and different terminologies used for surgical pathology. We aimed to evaluate correlations between US characteristics, cytologic results of FNAB, and thyroid antibodies, calcitonin, and thyroid function tests in patients presented with thyroid nodules, and to contribute in diagnosis, treatment, and patient follow-up.

Methods: A total of 1639 patients with thyroid nodules who applied to outpatient clinic of endocrinology between dates April and May 2017, had FNAB under US guideline, and their pathologic evaluation was performed according to Bethesda classification. Serological and hormonal tests were also performed for each patient.

Results: The mean age of study group was 50 (range interval=14-90) years. The median of nodediameter1 was 17.5 (range=1-51) $\mathrm{mm}$, and median of node-diameter2 was 12 (range=8-33) $\mathrm{mm}$. Of US characteristics, echogenicity, microcalcification, irregular borders, and solitary nodules were determined in $4.4 \%, 54 \%, 71.2 \%$, and $86.6 \%$ of cohort respectively. Elevated anti-TPO was determined in $64.6 \%$. Cytologic readings were reported as $15.8 \%$ nondiagnostic, $53.8 \%$ atypia of undetermined significance/follicular lesion of undetermined significance, $28.8 \%$ benign, $0.4 \%$ suspicious for follicular nodule, and $1.2 \%$ malign.

Conclusion: Measurement of thyroid autoantiboides, calcitonin, and thyroid function tests have good correlations with Bethesda classification in patients with thyroid nodules.
\end{abstract}

Key words: Thyroid Nodule; Fine Needle Aspiration; Bethesda System; Ultrasonography; Cytology.

\section{Introduction}

Fine needle aspiration biopsy (FNAB) has currently an essential role in screening, diagnosis and treatment of thyroid nodules. FNAB not only prevents unnecessary thyroid surgery for benign nodules, but also has increased malignancy rate in resected nodules from $14 \%$ to $50 \%$. $^{1}$ Although FNAB is currently a widely accepted screening procedure in diagnosis of thyroid nodules, there has been confusion related to diagnostic terminology in the assessment of samples. This confusion has been caused by multiple category names, descriptive reports without assigning to a category, and different terminologies used for surgical pathology. ${ }^{2}$ Bethesda system for reporting thyroid cytopathology has been evolved to facilitate effective communication among cytopathologists, endocrinologists, surgeons, and other healthcare providers. ${ }^{1}$

On the other hand professional associations and expert groups have not reached a consensus whether all patients with thyroid nodules should be screened. The American Thyroid Association (ATA) considers that available data are not sufficient to propose screening. The European Thyroid Association (ETA) consensus has stated measurement of serum calcitonin levels as the part of work-up for all thyroid nodules. The updated guideline of American Association of Clinical Endocrinologists (AACE), Americal College of Endocrinology (ACE), and the Associazione Medici Endocrinologi (AME) have recommended measurement of serum calcitonin, if there are suspicious ultrasound findings or undeterminate cytological findings. ${ }^{3}$ 
In 2016 guideline of ACCE, ACE, and AME the basis of thyroid nodule management has been stated as use of high resolution ultrasonography (US), sensitive thyrotropin assay, and FNAB. Measurement of thyroid antibodies has also been recommended according to initial laboratory results for thyroid function. ${ }^{4}$

In the present study, we aimed to evaluate correlations between US characteristics, cytologic results of FNAB, and thyroid antibodies, calcitonin, and thyroid function tests in patients presented with thyroid nodules, and to contribute in diagnosis, treatment, and patient follow-up.

\section{Materials and Methods}

A total of 1639 patients with thyroid nodules who applied to outpatient clinic of endocrinology between dates April and May 2017, had FNAB under US guideline, and their pathologic evaluation was performed according to Bethesda classification. Of patients, 1390 (84.8\%) were females, and 249 (15.2\%) were males. US characteristics, thyroid antibody and calcitonin levels, and thyroid function tests were recorded.

In US examination, all nodules were evaluated for exogenicity, micro-/macrocalcification, regularity of borderline, cystic or solid or mixed characteristics, and also being solitary or multiple.

FNAB was performed by using $23 \mathrm{G}$ needle with $10 \mathrm{ml}$ syringe. Air dried samples were stained by Giemsa stain, and wet ethanol fixed smears were stained with hemotoxylin and eosin. Cytologic diagnoses were classified as unsatisfactory, benign, atypia of undetermined significance (AUS)/ follicular lesion of undetermined significance (FLUS), suspicious for follicular nodule, and malign.

Thyroid function tests were performed on ???? (....,...). Cytomorphologic features were reviewed and graded according to lymphocyte infiltrates, and other parameters. Findings were analyzed with antithyroid peroxidase (anti-TPO) antibodies, and hypersensitive thyroglobulin.

This study was approved by the local ethics committee, and written informed consents of patients were proided after informing them about the study in detail.

\section{Statistical Analysis}

Statistical analysis was performed by using SPSS 24 version. After normality testing for each variable, Kolmogorov-Smirnov and Shapiro-Wilk tests were performed. As variables were $p<0.05$, it was determined that variables were distributed abnormally, so non-parametric methods were used for analysis. Available data were both categoric and continuous in character. Chi square test was used to determine correlation between two categoric data, Kendall's Tau-b correlation coefficient was used to analyze one continuous and one categoric variable. As normal distribution could not be provided, median and rank (min-max) values are given in descriptive statistics.

\section{Results}

The mean age of study group was 50 (range $=14-90)$ years, and $15.2 \%$ of the cohort $(n=249)$ was composed of males, whereas majority $(n=1390)$ was females. The median of node-diameter1 was 17.5 (range $=1-51)$ $\mathrm{mm}$, and median of node-diameter 2 was 12 (range $=8-33) \mathrm{mm}$. In US examination echogenicity was absent in 1567 patients, whereas microcalcifications were observed in 885subjects. Details of ultrasonographic examination are presented in Table 1. 
Table 1. Baseline characteristics and US results of patients

\begin{tabular}{cll} 
Gender & Frequency $(\mathbf{N})$ & Percentage $(\%)$ \\
\hline Male & 249 & 15.2 \\
Female & 1390 & 84.8 \\
Total & 1639 & 100.0
\end{tabular}

\section{Echogenicity}

$\begin{array}{lll}\text { Absent } & 1567 & 95.6 \\ \text { Present } & 72 & 4.4 \\ \text { Total } & 1639 & 100.0\end{array}$

Microcalcification

$\begin{array}{lll}\text { Absent } & 754 & 46.0 \\ \text { Present } & 885 & 54.0 \\ \text { Total } & 1639 & 100.0\end{array}$

\section{Macrocalcification}

$\begin{array}{lll}\text { Absent } & 980 & 59.8 \\ \text { Present } & 659 & 40.2 \\ \text { Total } & 1639 & 100.0\end{array}$

\section{Borderlines}

$\begin{array}{lll}\text { Irregular } & 1167 & 71.2 \\ \text { Regular } & 472 & 28.8 \\ \text { Total } & 1639 & 100.0\end{array}$

Cystic

$\begin{array}{lll}\text { No } & 1376 & 84.0 \\ \text { Yes } & 263 & 16.0 \\ \text { Total } & 1639 & 100.0\end{array}$

\section{Solid}

$\begin{array}{lll}\text { No } & 821 & 50.1\end{array}$

$\begin{array}{lll}\text { Yes } & 818 & 49.9\end{array}$

$\begin{array}{lll}\text { Total } & 1639 & 100.0\end{array}$

Mixed

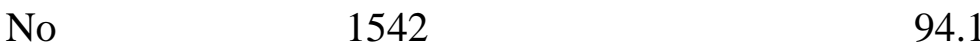

$\begin{array}{lll}\text { Yes } & 97 & 5.9\end{array}$

$\begin{array}{lll}\text { Total } & 1639 & 100.0\end{array}$

\section{Solitary nodule}

$\begin{array}{lll}\text { No } & 219 & 13.4 \\ \text { Yes } & 1420 & 86.6 \\ \text { Total } & 1639 & 100.0\end{array}$

Multiple nodules

$\begin{array}{lll}\text { No } & 1611 & 98.3 \\ \text { Yes } & 28 & 1.7 \\ \text { Total } & 1639 & 100.0\end{array}$


Serological and hormonal results indicated that $64.6 \%(\mathrm{n}=1058)$ of patients had anti-TPO $>34 \mathrm{IU} / \mathrm{ml}$ (Normal $=<9 \mathrm{IU} / \mathrm{ml}) ; 86 \%(\mathrm{n}=1409)$ of patients had anti-Tg $>115 \mathrm{IU} / \mathrm{ml}(\mathrm{Normal}=0-4 \mathrm{IU} / \mathrm{ml})$, and $1.3 \%$ of patients $(\mathrm{n}=22)$ had calcitonin level $>0-5 \mathrm{pg} / \mathrm{ml}($ Normal $=<0-5 \mathrm{pg} / \mathrm{ml})$ (Table 2). The mean TSH value was determined $4.43($ Normal $=0.4-0.3)($ range $=16.80-0.03) \mu \mathrm{lU} / \mathrm{ml}$.

Table 2. Serum levels of anti-TPO, Tg, and calcitonin in the study cohort

\begin{tabular}{lll}
\hline Anti-TPO & Frequency & Percentage \\
\hline$<34$ & 581 & 35.4 \\
$>34$ & 1058 & 64.6 \\
Total & 1639 & 100.0 \\
Anti-Tg & Frequency & Percentage \\
$<115$ & 230 & 14.0 \\
$>115$ & 1409 & 86.0 \\
Total & 1639 & 100.0 \\
Calcitonin & Frequency & Percentage \\
$<0-5 \mathrm{pg} / \mathrm{ml}$ & 1617 & 97.8 \\
$>0-5 \mathrm{pg} / \mathrm{ml}$ & 22 & 1.3 \\
Total & 1639 & 100.0 \\
\hline
\end{tabular}

TPO, Tyrosine peroxidase; Tg, Thyroglobulin.

As cytologic evaluation of nodules shown in Table 3, the majority of cohort (53.8\%) belonged to AUS/FLUS category.

Table 3. Cytologic results of nodules

\begin{tabular}{llllll}
\hline & \multicolumn{5}{c}{ Cytology category: No. of patients (\%) } \\
\cline { 2 - 6 } $\begin{array}{l}\text { Total No. } \\
\text { of Cases }\end{array}$ & Nondiagnostic & AUS/FLUS & Benign & $\begin{array}{l}\text { Suspicious } \\
\text { for FN }\end{array}$ & Malign \\
1639 & $259(15.8)$ & $882(53.8)$ & $472(28.8)$ & $6(0.4)$ & $20(1.2)$ \\
\hline
\end{tabular}

AUS, atypia of undetermined significance; FLUS, follicular lesion of undetermined significance; FN, follicular nodule

Gender was not deterministic and effective on Bethesda classification ( $p>0.05)$. There was $51.8 \%$ positive increasing and significant correlation between Bethesda classification and TSH $(\mathrm{p}<0.05)$. There was a negative weak correlation between Bethesda classification and node-diameter1 (decreasing effect of 6.4\%) $(\mathrm{p}=0.001)$. A positive and increasing correlation of $52.5 \%$ was determined between Bethesda classification and node-diameter2 $(\mathrm{p}=0.000)$.

Node-diameter1 variable had a positive significant increasing correlation of $8.3 \%$ with anti-TPO $(p=0.000)$, whereas it had no significant correlation with TSH, anti-Tg or calcitonin. Node-diameter2 variable had positive significant correlation of $44.7 \%$ with TSH $(\mathrm{p}=0.000)$, and of $16 \%$ with anti-TPO. There was no significant correlation between node-diameter 2 and anti-Tg or calcitonin.

\section{Discussion}

The results of this large sized thyroid FNA study confirmed and reinforced most of the common knowledge regarding the usefulness of this diagnostic tool. ${ }^{5,6}$ In this present study, FNAB results of 1639 thyroid nodules were distributed in 5 categories: $15.8 \%$ nondiagnostic specimen, $28.8 \%$ benign thyroid nodules, $53.8 \%$ AUS/FLUS, $0.4 \%$ suspicious for malignancy, and $1.2 \%$ positive for malignancy.

Similarly, Pathak et al. ${ }^{7}$ noted in their 4-category FNAB study that introduction of 6-category Bethesda System resulted in reducing number of non-diagnostic/undeterminate (AUS/FLUS) cases as compared to previously-used system, enabling better clinical usefulness of FNA results of thyroid gland. Richmond et al. ${ }^{8}$ reported that 68 out of 100 cases were defined as undeterminate according to previous system of categorization. However, they indicated that significant ratio of those AUS/FLUS cases were diagnosed as benign lesions after introduction of Bethesda system, and number of unnecessary surgery was decreased.

For nodules with the AUS/FLUS category further interventional options are repeatition of FNAB, diagnostic surgical excision, or surveillance, which are decided upon US findings, clinical risk factors, and preference of the individual patient. ${ }^{9}$ US examination differentiates simple cysts from solid nodules or from mixed cysts and solid nodules, which have 5\% malignancy potential. US characteristics such as hypoechogenicity, microcalcifications, irregular borders, and signs indicating invasion or regional lymphadenopathy are associated with cancer risk. ${ }^{10}$

In the literature, there are many studies which have investigated US characteristics to estimate the malignancy risk of thyroid nodules with AUS/FLUS results at initial cytology. ${ }^{11-15}$ In these studies, nodules with more than one 
suspicious US characteristic had a higher malignancy rate than nodules without any suspicious US characteristics. Also, calculated malignancy rate of nodules with more than one suspicious US characteristic ranged between $79.3 \%$ and $100 \% .{ }^{11-13,15}$ However, when cancer rates were calculated separately for AUS and FLUS nodules, the malignancy rates were different for AUS and FLUS nodules without suspicious US features (11.4\% and 3.9\%), and those with suspicious US features $(87 \%$ and $46.6 \%) .{ }^{14}$ Similar to the literature, majority of cytology readings were undeterminate (AUS/FLUS) in our cohort, and three of US characteristics (irregularity of borders, solitary nodules, and presence of microcalcifications) indicated that malignancy potential should be ruled out. In this sense, both undeterminate cytology readings and US findings were informative risk stratification data for clinicians.

In 2016 update guideline from the American Association of Clinical Endocrinologists, American College of Endocrinology and the Associazione Medici Endocrinologi on the diagnosis and management of thyroid nodules, experts recommended measurement of calcitonin levels if there were suspicious ultrasonographic findings or indetermined cytologic readings for early diagnosis of medullary thyroid carcinoma. ${ }^{4}$ In results of the study cohort, serum calcitonin levels were mildly elevated $(>0-5 \mathrm{pg} / \mathrm{ml})$ in $1.3 \%$ of patients. Absence of significant correlation between serum calcitonin and node-diameter 1 and diameter 2 was not surprising at mildly elevated levels, we believed that serum calcitonin measurement should be routinely measured in patients presenting with thyroid nodules.

Jeong et al. tried to establish malignancy rate in 165 thyroid nodules with AUS/FLUS cytology reading, and they used ultrasonographic characteristics and exhibition of BRAF V600E mutation. ${ }^{12}$ They reported that AUS/FLUS nodules were not only taller than wide shape, but also had irregular borders, and microcalcifications. ${ }^{12}$ According to mean of diameters $1 \& 2$, thyroid nodes had mainly oval shape, which was less related with malignancy (Odds Ratio: 1.000). ${ }^{12}$ De Micco et al. reported in their research in assessment of thyroid peroxidase (TPO) immunodetection in FNAB of thyroid nodules that sensitivity of TPO staining was $100 \%$ in malignancy, specificity was $90 \%$, overall accuracy of $92 \%$ with discrimination limit of $80 \%$ between benign and high-risk nodules. ${ }^{16}$ The authors concluded that TPO staining was a useful tool with FNAB cytology for thyroid nodules. ${ }^{16}$ In our cohort, both node-diameter1 and 2 had positive correlations with anti-TPO, but at low rates. We believed this was indicative for less malignancy rate in AUS/FLUS nodes.

The main limitation of our study is that we have not evaluated postoperative pathology reports of the patients according to cytology categorization and US findings. This would clarify interpretation of results more.

\section{Conclusion}

In conclusion, measurement of thyroid autoantiboides, calcitonin, and thyroid function tests have good correlations with Bethesda classification in patients with thyroid nodules.

\section{References}

[1] Cibas ES, Ali SZ. The Bethesda system for reporting thyroid cytopathology. Am J Clin Pathol 2009;132:658665.

[2] Wesola M, Jelen M. Bethesda system in evaluation of thyroid nodules: review. Adv Clin Exp Med 2017;26:177-82.

[3] Tormey WP, Byrne B, Hill AD, Sherlock M, Thompson CJ. Should serum calcitonin be routinely measured in patients presenting with thyroid nodule? Minerva Endocrinologica 2017;42:306-10.

[4] Gharib H, Papini E, Garber JR, Duick DS, Harrell RM, Hegedüs L, Paschke R, Valcavi R, Vitti P; AACE/ACE/AME Task Force on Thyroid Nodules. American Association of Clinical Endocrinologists, American College of Endocrinology, and Associazione Medici Endocrinologi medical guidelines for clinical practice for the diagnosis and management of thyroid nodules-2016 update. Endocr Pract. 2016;22:622-39.

[5] Amrikachi M. Ramzy I, Rubenfield S, Wheeler TM. Accuracy of fine-needle aspiration of thyroid: a review of 6226 cases and correlation with surgical or clinical outcome. Arch Pathol Lab Med. 2001;125:484-8.

[6] Wu HJ, Jones JN, Osman J. Fine-needle aspiration cytology of the thyroid: ten year experience in a community teaching hospital. Diagn Cytopathol. 2006;34:87-8.

[7] Pathak P, Srivastava R, Singh N, Arora VK, Bhatia A. Implementation of the Bethesda System for Reporting Thyroid Cytopathology: Interobserver concordance and reclassification of previously inconclusive aspirates (published online on April 1 2014). Diagn Cytopathol. 2014.

[8] Richmond BK, O'Brien BA, Mangano W, Thompson S, Kemper S. The Impact of implementation of the Bethesda System for Reporting Thyroid Cytopathology on the surgical treatment of thyroid nodules. Am Surg. 2012;78(6):706-10.

[9] Haugen BR, Alexander EK, Bible KC, et al. 2015 American Thyroid Association management guidelines for adult patients with thyroid nodules and differentiated thyroid cancer: the American Thyroid Association guidelines task force on thyroid nodules and differentiated thyroid cancer. Thyroid. 2016;26(1):1-133. 
[10] Hegedüs L. Thyroid ultrasound. Endocrinol Metab Clin North Am 2001;30:339-60.

[11] Gweon HM, Son EJ, Youk JH, Kim JA. Thyroid nodules with Bethesda system III cytology: can ultrasonography guide the next step? Ann Surg Oncol. 2013;20(9):3083-3088.

[12] Jeong SH, Hong HS, Lee EH, Cha JG, Park JS, Kwak JJ. Outcome of thyroid nodules characterized as atypia of undetermined significance or follicular lesion of undetermined significance and correlation with ultrasound features and BRAF(V600E) mutation analysis. Am J Roentgenol. 2013;201(6):W854-W860.

[13] Kim DW, Lee EJ, Jung SJ, Ryu JH, Kim YM. Role of sonographic diagnosis in managing Bethesda class III nodules. Am J Neuroradiol. 2011;32(11):2136-2141.

[14] Rosario PW. Thyroid nodules with atypia or follicular lesions of undetermined significance (Bethesda Category III): importance of ultrasonography and cytological subcategory.

Thyroid. 2014;24(7):1115-1120.

[15] Yoo WS, Choi HS, Cho SW, et al. The role of ultrasound findings in the management of thyroid nodules with atypia or follicular lesions of undetermined significance. Clin Endocrinol (Oxf). 2014;80(5):735-742.

[16] De Micco C, Zoro P, Garcia S, Skoog L, Tani EM, Carayon P, Henry JF. Thyroid peroxidase immunodetection as a tool to assist diagnosis of thyroid nodules on fine-needle aspiration biopsy. Eur $\mathrm{J}$ Endocrinol. 1994;131(5):474-9. 